\title{
Why the future of health care is salaried
}

\author{
- Cite as: CMAJ 2019 December 16;191:E1390-1. doi: 10.1503/cmaj.1095819
}

Posted on cmajnews.com on November 28, 2019

$\mathbf{F}$ ee-for-service doesn't work well for an aging population with chronic conditions, according to an expert panel tasked by the Alberta government to make recommendations on provincial spending. "A key factor driving physician costs is how physicians are paid," the panel reports. While fee-forservice works well in emergency rooms and surgical settings, "it is not suited to the primary health care model," the panel explains, recommending a move to alternative payment models.

Dr. Tara Kiran, a family physician and clinician investigator at St. Michael's Hospital, says fee-for-service has lost relevance as primary care has become more complex. "We're balancing many different evidence guidelines from different professional and nonprofit organizations ... and we're often balancing the evidence for people with multiple conditions," Kiran explains. "Doing that takes time," but fee-for-service payment models disincentivize doctors to take that time, she says.

According to Dr. Ruth Lavergne, an assistant professor in health services at Simon Fraser University, "there's widespread recognition that fee for service may not be well aligned with the goals of primary care or chronic disease management."

Lavergne agrees that fee-for-service schemes tend not to cover timeconsuming but important aspects of chronic care like connecting patients to community resources. And it can be difficult to incentivize doctors to manage a population of patients proactively if they're paid only for services provided to the individual in front of them.

While some provinces have introduced additional incentives for chronic care under fee-for-service plans, these haven't improved outcomes. Lavergne says that in British Columbia, for example, "the incentives didn't do anything to address the question of team-based care or other structural support needed."

Meanwhile, there is some evidence that alternative payment plans improve chronic disease management. Kiran led a study in Ontario that found doctors who were paid a set amount per personal enrolled in their practice were more likely to refer patients for recommended diabetes testing than those paid fee-for-service.

Internationally, many top performing health systems pay doctors a salary with some financial incentives for volume and quality of care, Kiran adds. And studies of accountable health care organizations in the United States, which incentivize doctors to work together to improve outcomes and save money, have shown modest improvements in indicators for cancer screening, pediatric care quality 
and chronic disease management. Those structured as a "medical home," where a doctor coordinates a team of health providers, demonstrated further quality improvements.

However, switching payment models too quickly, or without supports for collaborative care, can backfire. Health care researcher Dr. Emily Gard Marshall warns that Alberta will "risk losing physicians" if it follows the expert panel's advice to legislate alternative payments. She rec- ommends a softer rollout, along the lines of Nova Scotia's approach; that province created a range of alternative payment and health care delivery models and worked with physicians to ensure each fit local need. These included "turnkey" options, where the province provides a building and staff and pays physicians on salary, and more "informal" teams, where two or three fee-for-service doctors collaborate with a government-paid nurse practitioner.
According to Lavergne, British Columbia missed the mark earlier this year when it launched a new salarybased payment plan for physicians without investing in clinical information systems to connect practices to community resources. "Switching physicians overnight to capitation on its own wouldn't necessarily yield any changes," she says.

Wendy Glauser, Toronto, Ont. 